\title{
Disease burden and mental health system capacity: WHO Atlas study of 117 low- and middle-income countries
}

\author{
Ryan McBain, Carmel Salhi, Jodi E. Morris, Joshua A. Salomon and Theresa S. Betancourt
}

\section{Background}

Treatment coverage for mental disorders ranges from less than $10 \%$ to more than $90 \%$ across low- and middle-income (LAMI) countries. Studies have yet to examine whether the capacity of mental health systems might be adversely affected by the burdens of unrelated conditions such as HIV/AIDS.
Aims
To examine whether the magnitude of disease burden from communicable, perinatal, maternal and nutritional conditions - commonly referred to as Group 1 diseases - is inversely associated with mental health system capacity in LAMI countries.

\section{Method}
Multiple regression analyses were undertaken using data from 117 LAMI countries included in the 2011 World Health Organization (WHO) Mental Health Atlas. Capacity was

defined in terms of human resources and infrastructure. Regressions controlled for effects of political stability, government health expenditures, income inequality and neuropsychiatric disease burden.

\section{Results}

Higher Group 1 disease burden was associated with fewer psychiatrists, psychologists and nurses in the mental health sector, as well as reduced numbers of out-patient facilities and psychiatric beds in mental hospitals and general hospitals $(t=-2.06$ to $-7.68, P<0.05)$.

\section{Conclusions}

Evidence suggests that mental health system capacity in LAMI countries may be adversely affected by the magnitude of their Group 1 disease burden.

\section{Declaration of interest}

None.
An estimated 450 million people worldwide currently have at least one mental disorder. ${ }^{1}$ Although studies have shown that behavioural and pharmacological interventions are effective in treating symptoms of many of these disorders, ${ }^{2}$ the treatment gap - defined as the difference between the number of individuals with a disorder and the number actually treated - ranges from less than $10 \%$ to more than $90 \%$ across low- and middle-income (LAMI) countries. $^{3-5}$ This begs the question why some LAMI countries' mental health systems have dramatically greater capacity than others. ${ }^{6,7}$ Why, for example, do some countries have 1 psychiatrist for every 30000 citizens, while others have 1 for every 4 million? One hypothesis which remains untested is that countries' communicable, maternal, perinatal and nutritional conditions - commonly referred to as Group 1 diseases ${ }^{8}$ - compete for resources that might otherwise be allocated to mental healthcare. Group 1 diseases such as HIV/AIDS, tuberculosis and malaria represent a constellation of conditions that disproportionately affect morbidity and mortality levels in LAMI countries, as compared with high-income countries ${ }^{9}$; and this generates strong a priori reason to suspect that Group 1 diseases may undermine mental health system capacity in such settings. Competition for resources within countries' health systems has been consistently documented in areas outside of psychiatric services. ${ }^{10,11}$

In the present paper, we examine the relationship between Group 1 disease burden and key indicators of mental health system capacity, taking into account disparities in countries' economic development and neuropsychiatric burden of disease. We hypothesised that the magnitude of a country's Group 1 disease burden would be inversely associated with mental health system capacity. We also theorised that the impact of government expenditures on health - one aspect of economic development would be mediated by Group 1 disease burden, meaning that health expenditures are initially allocated to address Group 1 diseases, after which resources - including personnel and infrastructure - are targeted towards mental healthcare.

\section{Method}

\section{Sample}

The sample comprised 117 LAMI countries which were included in the 2011 WHO World Mental Health Atlas survey. ${ }^{7}$ In total, 134 LAMI countries completed the survey; however, 17 had a population under 0.5 million and were excluded from the analysis to avoid distortion in prevalence rates. Table 1 provides further descriptive statistics of the sample.

The WHO Mental Health Atlas represents the largest crossnational inventory of mental health resources currently available, documenting information across several mental health system domains, including legislation, financing, infrastructure and human resources. Although data have been collected in 2001, 2005 and 2011, changes in the operational definitions of indicators and survey items limit the ability to perform longitudinal data analysis.

Mental Health Atlas information is collected by country-based focal points, typically within Ministries of Health. Data predominately reflect country resources between the years 2008 and 2010. In order to facilitate accuracy and consistency, several steps were taken. First, WHO headquarters provided a glossary of operational definitions to ensure that all countries reported information on the same entities, institutions and human resources. Second, once country-based information was transferred to WHO headquarters, a series of validity checks were conducted, including the detection of outliers relative to other 


\begin{tabular}{|c|c|c|c|}
\hline World Health Organization region & $\begin{array}{l}\text { Median population, } \\
\text { millions (s.d.) }\end{array}$ & $\begin{array}{l}\text { Median US\$ GNI } \\
\text { per capita (s.d.) }\end{array}$ & $\begin{array}{l}\text { Median Group } 1 \text { DALYs } \\
\text { per } 100000 \text { population (s.d.) }\end{array}$ \\
\hline Africa $(n=43)$ & $1.2(2.8)$ & 680 (1972) & 27846 (11722) \\
\hline Americas $(n=21)$ & $1.0(4.6)$ & 4570 (2645) & 3995 (3693) \\
\hline Eastern Mediterranean $(n=14)$ & $2.7(4.8)$ & $2310(2108)$ & 4638 (10 470) \\
\hline European $(n=20)$ & $0.7(3.7)$ & 4770 (3011) & 2657 (2538) \\
\hline South-East Asia $(n=9)$ & $5.0(38.9)$ & 2005 (1075) & 10529 (3391) \\
\hline Western Pacific $(n=10)$ & $1.1(42.1)$ & 1405 (2109) & 5429 (5113) \\
\hline Total $(n=117)$ & $1.0(16.9)$ & $2050(2718)$ & $8628(13082)$ \\
\hline
\end{tabular}

countries of the same region and income bracket, as well as outliers relative to the information countries had previously provided in the 2000 and 2005 versions of the Atlas. Third, countries were provided with an opportunity to address mistakes, and if an adequate response was not offered, the data point was removed. A full overview of WHO Atlas data collection methodology can be found in the introduction of the 2011 edition. $^{7}$

\section{Dependent measures: mental health system capacity}

Mental health system capacity was defined in this study in terms of an array of key health system indicators across two core domains: human resources and facility infrastructure. Within the domain of human resources, the numbers of psychiatrists, nurses and psychologists per 100000 population working in the mental health sector were used. Psychiatrists are relatively rare in LAMI countries and are therefore required to assume multifaceted roles, variously diagnosing and treating patients, training staff and managing facilities. ${ }^{12}$ In contrast, nurses represent the largest group of employees in most LAMI countries' mental health sectors and are primarily responsible for the oversight and care of patients. ${ }^{7,13}$ Last, psychologists represent a human resource for which the main therapeutic focus is psychosocial (rather than pharmacological) intervention. ${ }^{14}$

Infrastructure was measured in terms of psychiatric beds per 100000 population in mental hospitals and general hospitals, as well as out-patient facilities per 100000 population. In the context of hospitals, the number of beds is considered to be a more precise indicator of capacity, as countries may have only a few facilities with many beds, or, conversely, many facilities with few beds. In contrast to hospitals, out-patient facilities typically serve patients on a diurnal basis, and therefore the number of facilities is more appropriate.

Availability of additional WHO World Health Statistics data ${ }^{15}$ allowed for the creation of three relative outcome measures of mental health system capacity: psychiatrists as a percentage of all physicians in the country, psychiatric beds in general hospitals as a percentage of all hospital beds, and psychiatric beds in mental hospitals as a percentage of all hospital beds. Although the primary dependent measures of interest represent overall (absolute) mental health system capacity, these three additional measures may be conceptualised as measures of relative capacity, i.e. the availability of resources in mental health as a function of countries' overall health systems.

\section{Independent measures}

\section{Disease burden}

Group 1 disease burden was quantified using age-standardised disability-adjusted life-years (DALYs) for 2004, as reported in the 2008 update of the WHO's Global Burden of Disease Project. ${ }^{9}$
Disability-adjusted life-years represent a health measure which unifies information on years of life lost due to premature mortality and years of life lived in disability. A full description of the statistical methods for deriving country estimates can be found in Annex B of the Global Burden of Disease 2004 update, ${ }^{9}$ but it should suffice to say that a broad range of United Nations (UN) data sources and estimation techniques, with a primary reliance on life tables from all UN member states, are central in this process. Accuracy of Group 1 disease burden estimates is contingent on the quality of countries' information systems, but for the primary contributors to DALYs in Group 1 - such as HIV/AIDS, malaria and diarrhoeal diseases - there are often longitudinal data that were used to inform final figures. As with information on mental health system capacity, any noise in these estimates would bias results towards the null hypothesis (i.e. non-significance).

Communicable, perinatal, maternal and nutritional diseases represent the primary (Group 1) disease cluster affecting LAMI countries and are reported in the WHO's global burden of disease (GBD) estimate as prevalence rates per 100000 population. ${ }^{16}$ For each country in the analysis, the aggregate value of the burden due to all conditions in this group (per 100000 population) was utilised. As a control measure, the number of DALYs attributable to neuropsychiatric conditions (as of 2004) was also included in analyses. This was utilised to account for the fact that, in countries with a more severe burden of neuropsychiatric conditions (e.g. as a consequence of war), the demand for mental health system capacity is likely to be greater. The inclusion of neuropsychiatric condition DALYs also accounts for possible reverse causation, whereby greater mental health system capacity reduces neuropsychiatric burden of disease and potentially affects resources available for treating Group 1 diseases.

\section{Economic measures}

Health system capacity is closely related to more general measures of a country's economic development. For example, wealthier countries typically allocate a higher percentage of their budget to healthcare. ${ }^{17}$ Conversely, poorer countries are more likely to have recently concluded a civil war, over the course of which infrastructure such as hospitals and community-based facilities are often compromised or intentionally destroyed. ${ }^{18}$

We selected three variables to capture economic disparities across countries. The first - government health expenditures per capita in 2006, ${ }^{15}$ measured in international dollars at purchasing power parity (PPP \$) - reflects both national-level income and resource allocation to health systems. On the one hand, if a fixed percentage of national-level income were directed to the health sector across all countries (e.g. 5\%), this would translate into greater government health expenditures in absolute terms for countries with a larger income per capita. However, as 
noted above, wealthier countries also tend to allocate a greater relative amount (percentage) of income to health. Thus, although income per capita alone could be used as a base measure of countries' economic development, utilising only the proportion directed to the health sector more accurately reflects financial resources available for developing health system capacity, which is the overarching outcome of interest in this study.

The second variable related to economic status was the 2006 Failed State Index, which is a multifaceted indicator of country governance and political stability, published yearly by the Fund for Peace. ${ }^{19}$ The confluence of poverty, social unrest and civil war can render governments incapacitated or unwilling to develop health system infrastructure; a country's Failed State Index score provides a unique vantage point for viewing this interaction.

The third economic variable used was the Gini coefficient, which measures income inequality. In most LAMI countries, income is positively skewed such that measures like income per capita insufficiently capture the average financial means of an individual. The Gini coefficient internalises this by indexing the heterogeneity (or statistical dispersion) of income within a country. Studies have shown that income inequality within and across countries is associated with greater prevalence of mental disorders. ${ }^{20,21}$ Gini coefficients are not available for all countries in every year; for our analysis we included the most recent value available for each country, which ranged between the years 2000 and 2010.

\section{Methods of analysis}

Ordinary least squares multiple linear regression analyses were conducted using STATA/SE 12.0 for Windows. The first model included only Group 1 disease burden as an explanatory variable. A second model included only economic measures in order to characterise the unadjusted relationship between overall country development and mental health system capacity. In the third step, both sets of independent variables were entered alongside DALYs from neuropsychiatric conditions, in order to account simultaneously for the range of possible predictors of mental health system indicators. Variables, apart from the Failed State Index, were highly positively skewed and were therefore log-transformed.

Missing data were addressed using a multiple imputation approach with 100 simulations. This approach characterises the joint probability distribution of all of the variables in the dataset to create multiple simulated values for missing observations, thereby reducing bias in addition to accounting for sampling variability across imputations. ${ }^{22}$ Although in most instances missing data were minimal, for four measures missingness was greater than 10\%: the Failed State Index (11.1\%), the number of nurses working in the mental health sector $(12.0 \%)$, the number of psychologists working in the mental health sector (12.8\%), and the number of psychiatric beds in general hospitals $(19.7 \%)$. In secondary analyses measuring relative outcomes, missingness was more substantial: $7.7 \%$ for the proportion of all physicians working as psychiatrists, $18.8 \%$ for the proportion of all hospital beds that are psychiatric beds in mental hospitals, and $29.9 \%$ for the proportion of all hospital beds that are psychiatric beds in general hospitals. To evaluate the impact of missingness and skewedness on point estimates and confidence intervals, regression analyses were also run on a non-imputed data-set using a non-parametric bootstrap (1000 repetitions), which allows for a bias adjustment in confidence intervals. Parameter estimates from this were comparable to those reported below using ordinary least squares with multiply imputed data-sets.
The second aspect of data analysis - mediation analysis - was conducted in MPlus 6.12 for Windows using path analysis. Of specific interest was the extent to which the relationship between government health expenditures and mental health system capacity was mediated by the magnitude of countries' Group 1 disease burden, represented by the pathway: health expenditures $\rightarrow$ Group 1 disease burden $\rightarrow$ mental health system capacity. Parameter estimates and confidence intervals were bias adjusted using a non-parametric bootstrap. Missing data were addressed in MPlus using the full information maximum likelihood approach.

\section{Results}

The median magnitude of Group 1 disease burden across countries was 8628 DALYs per 100000 population (s.d. $=13082$ ). Tables 2 and 3 present results from the multiple regression analyses assessing the relationships between the magnitude of country Group 1 disease burden and mental health system measures. In naive univariate regressions (Model 1), higher Group 1 disease burden was inversely associated with all outcome measures $(t=-4.82$ to -17.71 , $P<0.001)$. After controlling for measures of economic development and neuropsychiatric disease burden (Model 3), effect sizes were reduced but hypothesis test results remained significant $(t=-2.06$ to $-7.68, P<0.05)$ in all six instances. A $1 \%$ lower burden predicted $1.0 \%$ more psychiatrists $(\beta=-1.04$, $t=-7.68, P<0.001), 0.8 \%$ more nurses $(\beta=-0.79, t=-3.62$, $P<0.001)$ and $0.7 \%$ more psychologists $(\beta=-0.72, t=-3.94$, $P<0.001)$ in the mental health sector, as well as $0.5 \%$ more mental health out-patient facilities ( $\beta=-0.54, t=-2.61, P<0.05), 0.6 \%$ more psychiatric beds in mental hospitals $(\beta=-0.60, t=-2.06$, $P<0.05)$ and $0.6 \%$ more beds in general hospitals $(\beta=-0.57$, $t=-2.22, P<0.05)$. Figure 1 illustrates the relationship between Group 1 disease burden and number of psychiatrists working in the mental health sector.

Median health expenditures per capita (2006) across countries was PPP \$86 (s.d.=\$169). The average Gini coefficient - for which 0 indicates perfect equality of income and 100 indicates the highest level of inequality - was $42($ s.d. $=9)$. Similarly, the average Failed State Index score (lower is better) was 83 (range 23-113; s.d.=16). Among these three measures of economic development, health expenditure per capita was the most consistently significant across regression models: in the model containing only economic measures (Table 2, Model 2), health expenditure per capita was a significant predictor of all six outcomes ( $t=2.89$ to $9.16, P=0.01$ to $P<0.001)$. In the full model (Table 2, Model 3 ), health expenditures significantly predicted all human resources outcomes as well as the number of psychiatric beds in mental hospitals ( $t=2.36$ to $3.60, P=0.02$ to $P<0.001$ ); for the remaining outcomes this predictor was non-significant $(P>0.05)$.

Group 1 burden of disease was inversely associated with all three relative outcome measures: percentage of physicians that are psychiatrists, percentage of hospital beds that are psychiatric beds in mental hospitals and percentage of hospital beds that are psychiatric beds in general hospitals. However, in each case, the association was non-significant $(t=-0.02$ to -0.92 , $P=0.36$ to $P=0.99$ ).

Table 4 summarises the results from the mediation analyses. The magnitude of Group 1 disease burden mediated the relationship between government health expenditures and mental health system capacity for five of six outcomes, meaning that in all cases but one the indirect effect (health expenditures $\rightarrow$ Group 1 disease burden $\rightarrow$ mental health capacity) was significant ( $t=2.07$ to 5.00 , $P<0.05)$. The magnitude of the mediation effect - calculated as 


\begin{tabular}{|c|c|c|c|}
\hline Human resource & Model $1, \beta$ (s.e.) & Model $2, \beta$ (s.e.) & Model 3, $\beta$ (s.e.) \\
\hline $\begin{array}{l}\text { Number of psychiatrists in mental } \\
\text { Group } 1 \text { disease burden }{ }^{a} \\
\text { Government health expenditures } \\
\text { Failed State Index score } \\
\text { Income inequality (Gini) } \\
\text { Neuropsychiatric DALYs }{ }^{c} \\
\text { Model } R^{2}\end{array}$ & $-1.513(0.085)^{* * *}$ & $\begin{array}{c}0.995(0.109)^{* * *} \\
-0.018(0.010) \\
-0.044(0.013)^{\star *} \\
R^{2}=0.62\end{array}$ & $\begin{array}{r}-1.038(0.135)^{* * *} \\
0.378(0.105)^{* * *} \\
-0.006(0.007) \\
-0.003(0.011) \\
1.197(0.66) \\
R^{2}=0.79\end{array}$ \\
\hline $\begin{array}{l}\text { Number of nurses in mental health } \\
\text { Group } 1 \text { disease burden } \\
\text { Government health expenditures }^{\text {b }} \\
\text { Failed State Index score } \\
\text { Income inequality (Gini) } \\
\text { Neuropsychiatric DALYS } \\
\text { Model } R^{2}\end{array}$ & $-1.097(0.133)^{\star \star *}$ & $\begin{array}{c}0.743(0.133)^{* * *} \\
-0.018(0.013) \\
-0.032(0.016)^{*} \\
R^{2}=0.39\end{array}$ & $\begin{array}{c}-0.792(-0.219)^{* * *} \\
0.398(0.168)^{*} \\
-0.008(0.013) \\
-0.004(0.017) \\
-1.356(1.057) \\
R^{2}=0.47\end{array}$ \\
\hline $\begin{array}{l}\text { Number of psychologists in mental } \\
\text { Group } 1 \text { disease burden } \\
\text { Government health expenditures }^{\text {b }} \\
\text { Failed State Index score } \\
\text { Income inequality (Gini) } \\
\text { Neuropsychiatric DALYs } \\
\text { Model } R^{2}\end{array}$ & $-1.223(0.127)^{\star \star \star}$ & $\begin{array}{c}0.978(0.120)^{* * *} \\
-0.012(0.010) \\
0.020(0.014) \\
R^{2}=0.59\end{array}$ & $\begin{array}{c}-0.718(0.182)^{* * *} \\
0.545(0.144)^{* * *} \\
-0.004(0.010) \\
0.049(0.015)^{\star *} \\
0.928(0.849) \\
R^{2}=0.67\end{array}$ \\
\hline \multicolumn{4}{|c|}{$\begin{array}{l}{ }^{*} P<0.05, * * P<0.01, * * * P<0.001 . \\
\text { DALYS, disability adjusted life years. } \\
\text { a. Log of age-standardised DALYS per } 100000 \text { population attributable to communicable, perinatal, maternal and nutritional conditions. } \\
\text { b. Log of government health expenditure per capita in international dollars at purchasing power parity. } \\
\text { c. Log of age-standardised DALYS per } 100000 \text { population attributable to neuropsychiatric conditions. All mental health system capacity measures are log of prevalence per } 100000 \\
\text { population. }\end{array}$} \\
\hline
\end{tabular}

Table 3 Multiple regression analyses: infrastructure for mental healthcare

\begin{tabular}{|c|c|c|c|}
\hline Infrastructure & Model 1, $\beta$ (s.e.) & Model $2, \beta$ (s.e.) & Model $3, \beta$ (s.e.) \\
\hline $\begin{array}{l}\text { Number of psychiatric beds in general hospitals } \\
\text { Group } 1 \text { disease burden } \\
\text { Government health expenditures }^{b} \\
\text { Failed State Index score } \\
\text { Income inequality (Gini) } \\
\text { Neuropsychiatric DALYS } \\
\text { Model } R^{2}\end{array}$ & $-0.776(0.161)^{\star * *}$ & $\begin{aligned} & 0.504(0.175)^{* *} \\
-0.024(0.014) & \\
-0.036(0.020) & \\
R^{2}= & 0.25\end{aligned}$ & $\begin{array}{c}-0.571(0.257)^{\star} \\
0.370(0.218) \\
-0.013(0.014) \\
-0.020(0.021) \\
-3.062(1.211)^{*} \\
R^{2}=0.31\end{array}$ \\
\hline $\begin{array}{l}\text { Number of psychiatric beds in mental hospitals } \\
\text { Group } 1 \text { disease burden } \\
\text { Government health expenditures }^{b} \\
\text { Failed State Index score } \\
\text { Income inequality (Gini) } \\
\text { Neuropsychiatric DALYS } \\
\text { Model } R^{2}\end{array}$ & $-1.250(0.172)^{\star * *}$ & $\begin{array}{l}0.962(0.178)^{* * *} \\
-0.010(0.015) \\
-0.071(0.021)^{* *} \\
R^{2}=0.37\end{array}$ & $\begin{array}{c}-0.601(0.292)^{*} \\
0.654(0.231)^{*} \\
-0.003(0.015) \\
-0.049(0.023)^{*} \\
-0.179(1.342) \\
R^{2}=0.39\end{array}$ \\
\hline $\begin{array}{l}\text { Number of mental health out-patient facilities } \\
\text { Group } 1 \text { disease burden } \\
\text { Government health expenditures }^{\text {b }} \\
\text { Failed State Index score } \\
\text { Income inequality (Gini) } \\
\text { Neuropsychiatric DALYS } \\
\text { Model } R^{2}\end{array}$ & $-0.873(0.124)^{* * *}$ & $\begin{array}{l}0.482(0.124)^{* \star *} \\
-0.031(0.011)^{\star *} \\
-0.004(0.015) \\
R^{2}=0.37\end{array}$ & $\begin{array}{c}-0.537(0.206)^{*} \\
0.194(0.155) \\
-0.025(0.011)^{*} \\
0.016(0.016) \\
0.042(0.970) \\
R^{2}=0.42\end{array}$ \\
\hline $\begin{array}{l}{ }^{*} P<0.05, * * P<0.01, * * * P<0.001 . \\
\text { DALYS, disability adjusted life years. } \\
\text { a. Log of age-standardised DALYS per } 100000 \text { populatio } \\
\text { b. Log of government health expenditure per capita in ir } \\
\text { C. Log of age-standardised DALYS per } 100000 \text { populatio } \\
\text { population. }\end{array}$ & $\begin{array}{l}\text { nmunicable, perinatal, } \\
\text { at purchasing power } \mathrm{p} \\
\text { uropsychiatric conditio }\end{array}$ & $\begin{array}{l}\text { nutritional conditions. } \\
\text { nealth system capacity }\end{array}$ & of prevalence per 100000 \\
\hline
\end{tabular}

the indirect effect as a percentage of the total effect - ranged from $41.1 \%$ to $59.8 \%$.

\section{Discussion}

This is the first study to empirically assess the strength of relationships between communicable, perinatal, maternal and nutritional disease burden, economic development and mental health system capacity in a large number of LAMI countries. We found that the magnitude of Group 1 disease burden significantly predicts a number of key mental health system indicators, even after controlling for measures of nationallevel health expenditures, income inequality and political stability. Moreover, Group 1 disease burden mediates much of the relationship between health expenditures and mental health system capacity. 


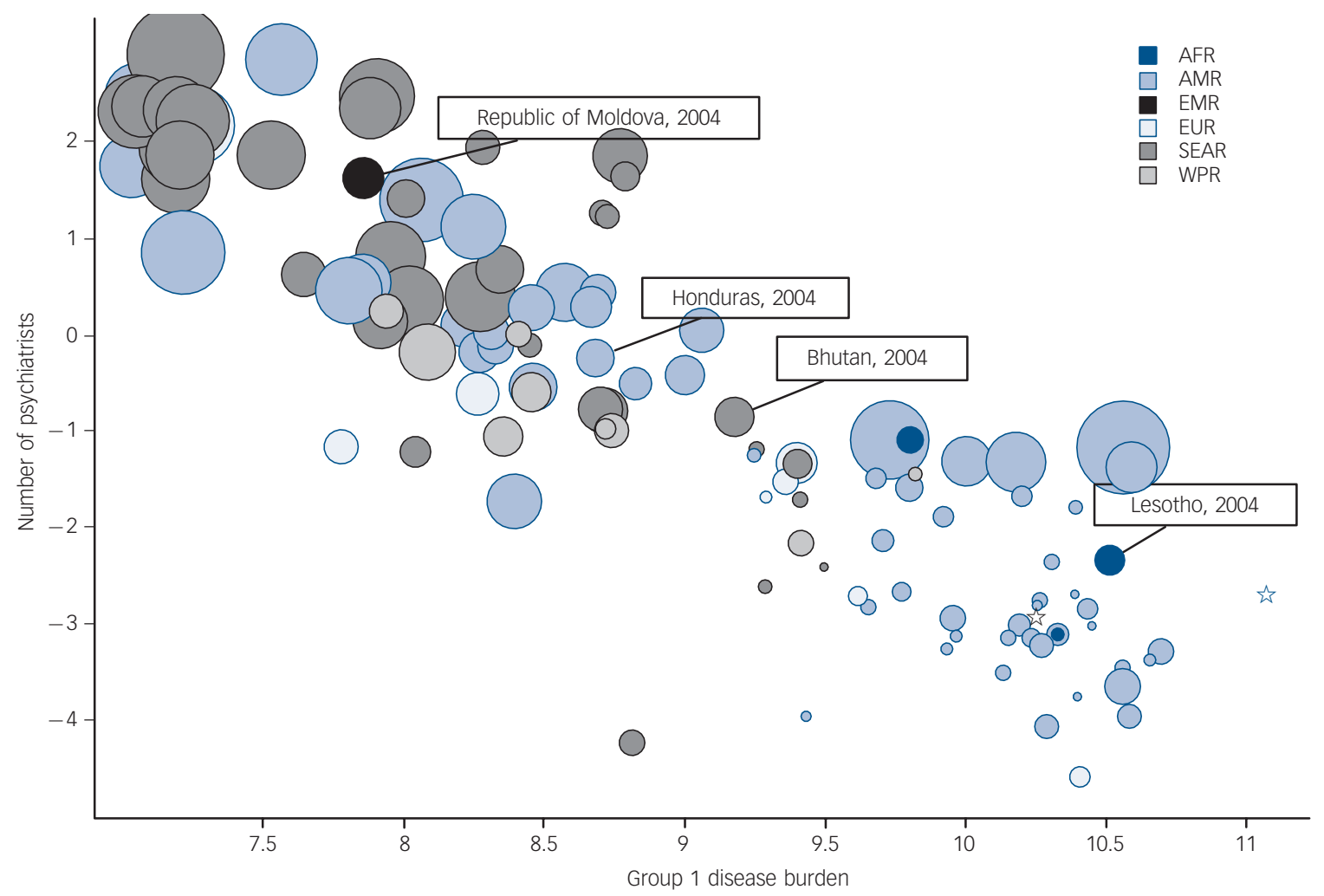

Fig. 1 Association between Group 1 disease burden and number of psychiatrists

The size of each bubble represents government health expenditures per capita, while colour corresponds to World Health Organization geographic region. Numbers of psychiatrists in the mental health sector are represented as the natural log of prevalence rates per 100000 population. Group 1 disease burden represents the natural log of age-standardised disability-adjusted life-years per 100000 population. The four highlighted countries serve to illustrate that, even when comparing countries with similar levels of health expenditures, the magnitude of disease burden is inversely associated with mental health system capacity for measures such as the number of psychiatrists. AFR, African Region; AMR, Americas Region; EMR, Eastern Mediterranean Region; EUR, European Region; SEAR, South-East Asia Region; WPR, Western Pacific Region.

\section{Mental health systems}

\section{and Group 1 disease burden}

The relationship between country burden of disease and mental health systems has rarely been discussed or evaluated in the literature. ${ }^{23}$ Our analysis shows that the magnitude of Group 1 disease burden is inversely associated with countries' mental health system capacity. Within the domain of human resources, for example, a $1 \%$ lower Group 1 disease burden predicted $1.0 \%$ more psychiatrists and $0.8 \%$ more nurses. By way of illustration, this implies that - compared with a country like Nigeria which has a Group 1 disease burden of almost 30000 DALYs per 100000 population - a similar country with half the disease burden (15000 DALYs) would be expected to have roughly twice as many psychiatrists and 1.8 times as many nurses working in the mental health sector. Similar observations are found in terms of health system infrastructure, including psychiatric beds in hospitals and out-patient facilities.

\section{Global burden of disease and resource allocation}

In settings where Group 1 burden of disease is large, neuropsychiatric conditions represent a smaller proportion of total disease burden and must compete with a wider array of health priorities. ${ }^{16}$ Although evidence shows that mental health interventions are relatively cost-effective - meaning that the cost per DALY averted is less than one to three times the national income per capita ${ }^{23}$ - they have less attractive cost-effectiveness ratios than interventions for many Group 1 disease conditions. For instance,

Table 4 Mediation analyses: Group 1 disease burden as a mediator of the relationship between government health expenditures and mental health systems capacity

\begin{tabular}{|c|c|c|c|}
\hline & Total effect $(95 \% \mathrm{Cl})$ & Indirect effect (95\% Cl) & $\%$ mediated \\
\hline \multicolumn{4}{|l|}{ Domain 1: human resources } \\
\hline Number of psychiatrists & $0.92(0.09)^{\star \star *}$ & $0.55(0.11)^{\star \star \star}$ & 59.8 \\
\hline Number of nurses in the mental health sector & $0.78(0.16)^{* * *}$ & $0.46(0.14)^{* *}$ & 59.0 \\
\hline Number of psychologists & $0.95(0.11)^{\star * *}$ & $0.39(0.10)^{\star \star *}$ & 41.1 \\
\hline \multicolumn{4}{|l|}{ Domain 2: infrastructure } \\
\hline Number of psychiatric beds in general hospitals & $0.59(0.18)^{\star *}$ & $0.31(0.15)^{\star}$ & 52.5 \\
\hline Number of psychiatric beds in mental hospitals & $1.01(0.19)^{* * *}$ & $0.30(0.18)$ & $N A b$ \\
\hline Number of mental health out-patient facilities & $0.49(0.12)^{\star \star *}$ & $0.27(0.12)^{*}$ & 55.1 \\
\hline \multicolumn{4}{|c|}{$\begin{array}{l}{ }^{*} P<0.05,{ }^{* * P}<0.01,{ }^{* * * P} P<0.001 \text {. } \\
\text { a. All mental health system capacity measures are prevalence per } 100000 \text { population. } \\
\text { b. Not applicable because total effect or indirect effect was non-significant at } P<0.10 \text {. }\end{array}$} \\
\hline
\end{tabular}


expanding immunisation coverage with standard child vaccines can cost PPP \$2-20 per DALY averted, ${ }^{24}$ whereas tricyclic antidepressants and psychosocial treatment for depression and anxiety - one of the most cost-effective mental health interventions - are estimated to cost more than PPP \$300 per DALY averted. ${ }^{2}$

Although we do not expect that decision makers explicitly allocate resources based solely on cost-effectiveness estimates, our findings suggest that there is an implicit recognition of these differences in the decision-making process. This has two implications: first, as LAMI countries experience variants of the epidemiological transition - particularly those in which the incidence of communicable disease declines over time $e^{25}-$ it is conceivable that countries will have greater resources to allocate to mental health. Second, in contexts where Group 1 disease burden is substantial, more persuasive arguments and focused donor support are needed to improve mental health system capacity. On the one hand, the mental health agenda setting process has made significant headway in recent years through initiatives such as The Lancet's series on global mental health (2007 and 2011; www.thelancet.com/series/) and WHO's Mental Health Gap Action Programme (www.who.int/mental_health/ mhgap/en/). On the other hand, data on the cost-effectiveness of mental health interventions in low-resource settings are limited and, until recently, have failed to account for positive externalities associated with interventions, including poverty reduction ${ }^{26}$ and reduced burden of care on family members. ${ }^{27,28}$ Given that as much as $50 \%$ of mental disorders develop during adolescence, and that these conditions often persist throughout the course of one's adult life, ${ }^{29}$ the spillover effects on family members' quality of life are likely to be substantial. Additionally, improvements in mental health are liable to affect one's physical health. For instance, studies have shown that individuals with affective disorders exhibit poor adherence to antiretroviral therapy $^{30}$ and are more likely to take up routine smoking habits. ${ }^{31}$

\section{Mediation analyses}

Results from mediation analyses in this study indicate that whether higher-income countries allocate their health budget to mental health systems is partly a function of existing disease burden: for five of six mental health system indicators, this mediatory role was significant $(P<0.05)$ and accounted for an average of $54 \%$ of the relationship between government health expenditures and mental health system capacity. Cumulatively, these findings support the hypothesis that whether financial resources are channelled to mental health is contingent on an indirect process through which Group 1 diseases are first targeted, after which available resources (including personnel and infrastructure) are shifted to address mental healthcare. For example, in Mozambique where government health expenditures are PPP \$26 per person per year and the Group 1 disease burden is 27800 DALYs per 100000 population, there is only 1 nurse in the mental health sector for every 300000 persons. In contrast, in Ghana, which has the same government health expenditures but a lower Group 1 disease burden of 17500 DALYs per 100000 population, the prevalence rate of nurses is seven times greater.

This conclusion has several ramifications. First, greater government expenditures on health do not necessarily translate directly into mental health system capacity; rather, it is the dynamics of and relationship between disease burden and level of health expenditures that affect the robustness of the mental health system. Second, and relatedly, insofar as gains in health expenditures translate into reductions in Group 1 disease burden, this may in the short-term do little to improve mental health outcomes, while in the long-run it may free up resources for expansion of the mental health agenda. Last, the development of policies and legislation intended to accelerate countries' mental health programmes should seek to work in conjunction with rather than in competition with - initiatives aimed at tackling Group 1 diseases. This synergistic approach to mental healthcare has been increasingly emphasised in low-resource settings. ${ }^{32}$

\section{Mental health and overarching health system capacity}

Findings from this study's relative outcome measures raise a question as to whether Group 1 disease burden uniquely affects mental health system capacity, or is reflective of a more general effect on countries' health systems. If we were to take the nonsignificance of these results as conclusive, then the data would indicate that substantial Group 1 disease burden overwhelms human resources and infrastructure of countries' overarching health systems, not just the mental health sector. However, given the substantial level of missingness of data associated with these relative measures, that all coefficients were negative (albeit nonsignificant), and that separate time points and methods of data collection were used for the WHO Mental Health Atlas and WHO World Health Statistics, further evidence is warranted before drawing any inferences.

\section{Limitations and further considerations}

An important limitation in this discussion is the use of crosssectional data. Here, several points should be noted. First, at a theoretical level, it is unlikely that improved mental health system capacity causes a decrease in Group 1 disease conditions. In contrast, a more direct argument can be made that lower rates of Group 1 diseases free up resources to develop mental health system capacity. Nevertheless, the possibility of reverse causality was partially addressed in our analysis by (a) the inclusion of DALYs attributable to neuropsychiatric conditions in regression models, and (b) the fact that global burden of disease estimates were measured at an earlier time point (2004) than outcomes (2011). Second, in final regression models the relationships between independent variables and outcomes were assessed after controlling for potential confounders, thereby reducing potential omitted variable bias. Third, the observed direction, magnitude and consistency of relationships among predictors and outcomes match well with a priori expectations, lending positive predictive validity to the findings.

That said, in several instances, results were close to significant but did not exceed the 0.05 alpha threshold. This may be a reflection of the limited power inherent in cross-national comparisons, insofar as these studies utilise data acquired through divergent means and at different points in time. In the present study, all outcome variables were ascertained through the 2011 Mental Health Atlas, and thus the comprehensive nature of this newly released WHO instrument is a strength. The heterogeneity in data collection methods and year of data collection among covariate measures reduces the reliability of specific point estimates reported in the manuscript; however, these inconsistencies are non-differential in nature, and we would therefore expect this to result in an attenuation bias - i.e. a bias towards non-significance. Given that we found a consistent, statistically significant set of results across outcome measures, this is of lesser concern. Last, it should also be noted that the majority of outcome indicators are institutionally oriented and provide limited commentary on the quality of care afforded by available capacity. 
In conclusion, this study represents a novel framework for viewing mental health system capacity in a diverse sample of LAMI countries. Subject to the constraints of existing data, this paper provides a set of consistent findings that communicable, perinatal, maternal and nutritional disease burden plays an central role in shaping mental health system capacity in LAMI countries, and that the magnitude of this burden mediates the relationship between government health expenditures and mental health system capacity in these settings. Moving forwards, research and policy efforts aimed at developing the global mental health agenda should take into account the dynamics of these relationships.

Ryan McBain, Carmel Salhi, Department of Global Health and Population, Harvard School of Public Health, Boston, Massachusetts, USA Jodi E. Morris, Department of Mental Health and Substance Abuse, World Health Organization, Geneva, Switzerland, Mental Health and Substance Abuse, World Health Organization, Geneva, Switzerland,
and School of Health and Exercise Sciences, University of British Columbia, Okanagan Campus, Kelowna, Canada; Joshua A. Salomon, Department of Global Health and Population, Harvard School of Public Health, Boston, Massachusetts, USA, Theresa S. Betancourt, Department of Global Health and Population, Harvard School of Public Health, and Research Program on Children and Global Adversity, FXB Center for Health and Human Rights, Harvard University, Boston, Massachusetts, USA

Correspondence: Ryan McBain, Harvard School of Public Health, 65 Huntington Avenue, Boston, MA 02115, USA. Email: rmcbain@hsph.harvard.edu

First received 26 Mar 2012, final revision 21 Jul 2012, accepted 3 Oct 2012

\section{Acknowledgements}

We would like to thank Dr Till Bärnighausen, Dr Sebastian Vollmer, Ms Chantelle Boudreaux and Mr Daniel Norton for their conceptual contributions to the manuscript. Additionally, WHO World Mental Health Atlas data would not be available if it were not for the the globe; a full acknowledgement of all individuals can be found in the 'Participating Countries and Contributors' section of the WHO's Mental Health Atlas 2011.

\section{References}

1 World Health Organization. The World Health Report 2001 - Mental Health: New Understanding, New Hope. WHO, 2001.

2 Patel V, Araya R, Chatterjee S, Chisholm D, Cohen A, De Silva M, et al Treatment and prevention of mental disorders in low-income and middleincome countries. Lancet 2007; 370: 991-1005.

3 Saxena S, Thornicroft G, Knapp M, Whiteford H. Resources for mental health: scarcity, inequity, and inefficiency. Lancet 2007; 370: 878-89.

4 Lora A, Kohn R, Levav I, McBain R, Morris J, Saxena S. Accessibility, patterns of care and the treatment gap in schizophrenic disorders: a WHO-AIMS survey in 50 low- and middle-income countries. Bull World Health Organ 2012; 90: 47-54.

5 Demyttenaere K, Bruffaerts R, Posada-Villa J, Gasquet I, Kovess V, Lepine JP et al. Prevalence, severity, and unmet need for treatment of menta disorders in the World Health Organization World Mental Health Surveys. JAMA 2004; 291: 2581-90

6 Saxena S, Sharan P, Garrido M, Saraceno B. World Health Organization's Mental Health Atlas 2005: implications for policy development. World Psychiatry 2006; 5: 179-84.

7 World Health Organization. Mental Health Atlas 2011. WHO, 2011.
8 Murray CJ, Lopez AD. Global mortality, disability, and the contribution of risk factors: Global Burden of Disease Study. Lancet 1997; 349: 1436-42.

9 World Health Organization. The Global Burden of Disease - 2004 Update. WHO, 2008.

10 Frenk J, Bobadilla JL, Sepulveda J, Cervantes ML. Health transition in middleincome countries: new challenges for healthcare. Health Policy Plann 1989; 4: 29-39.

11 World Health Organization. Everybody's Business: Strengthening Health Systems to Improve Health Outcomes. WHO, 2007.

12 Patel V. The future of psychiatry in low- and middle-income countries. Psychol Med 2009; 39: 1759-62.

13 World Health Organization. Atlas: Nurses in Mental Health 2007. WHO, 2007.

14 Kakuma R, Minas H, van Ginneken N, Dal Poz MR, Desiraju K, Morris JE, et al. Human resources for mental healthcare: current situation and strategies for action. Lancet 2011; 378: 1654-63.

15 World Health Organization. World Health Statistics. WHO, 2010.

16 Prince M, Patel V, Saxena S, Maj M, Maselko J, Phillips M, et al. No health without mental health. Lancet 2007; 370: 859-77.

17 Newhouse JP. Medical-care expenditure: a cross-national survey. J Hum Resour 1977; 12: 115-25.

18 Collier P. The traps. In The Bottom Billion: 53-64. Oxford University Press, 2007.

19 Fund for Peace. Failed State Index. Fund for Peace, 2011.

20 Pickett KE, James OW, Wilkinson RG. Income inequality and the prevalence of mental illness: a preliminary international analysis. J Epidemiol Community Health 2006; 60: 646-7.

21 Weich S, Lewis G, Jenkins SP. Income inequality and the prevalence of common mental disorders in Britain. Br J Psychiatry 2001; 178: 222-7.

22 Rubin DB. Multiple Imputation for Nonresponse in Surveys. Wiley, 1987.

23 World Health Organization. Dollars, DALYS and Decisions: Economic Aspects of the Mental Health System. WHO, 2006.

24 Laxminarayan R, Chow J, Shahid-Salles SA. Intervention cost-effectiveness: overview of main messages. In Disease Control Priorities in Developing Countries (eds DT Jamison, JG Breman, AR Measham, G Alleyne, M Claeson, DB Evans, et al.): 3-34. Oxford University Press, 2006.

25 Omran AR. The epidemiologic transition: a theory of the epidemiology of population change. Milbank Mem Fund Q 1971; 49: 509-38.

26 Lund C, De Silva M, Plagerson S, Cooper S, Chisholm D, Das J, et al. Poverty and mental disorders: breaking the cycle in low-income and middle-income countries. Lancet 2011; 378: 1502-14.

27 Dixon A, McDaid D, Knapp M, Curran C. Financing mental health services in low- and middle-income countries. Health Policy Plann 2006; 21: 171-82.

28 Caqueo-Urizar A, Gutierrez-Maldonado J. Burden of care in families of patients with schizophrenia. Qual Life Res 2006; 15: 719-24.

29 Belfer ML. Child and adolescent mental disorders: the magnitude of the problem across the globe. J Child Psychol Psychiatry 2008; 49: 226-36.

30 Ammassari A, Antinori A, Aloisi MS, Trotta MP, Murri R, Bartoli L, et al. Depressive symptoms, neurocognitive impairment, and adherence to highly active antiretroviral therapy among HIV-infected persons. Psychosomatics 2004; 45: 394-402.

31 Breslau N, Novak SP, Kessler RC. Psychiatric disorders and stages of smoking. Biol Psychiatry 2004; 55: 69-76.

32 Dua T, Barbui C, Clark N, Fleischmann A, Poznyak V, van Ommeren M, et al. Evidence-based guidelines for mental, neurological, and substance use disorders in low- and middle-income countries: summary of WHO recommendations. PLOS Med 2011; 8: e1001122. 